\title{
Rectal Lymphoma: A Diagnostic Challenge
}

\author{
Koh Ging Wong \\ Department of Surgery, Sibu General Hospital, Sibu, Malaysia \\ Email: wongkohging@gmail.com
}

Received 1 March 2015; accepted 31 March 2015; published 9 April 2015

Copyright (C) 2015 by author and Scientific Research Publishing Inc.

This work is licensed under the Creative Commons Attribution International License (CC BY).

http://creativecommons.org/licenses/by/4.0/

(c) (i) Open Access

\begin{abstract}
Rectal lymphoma is a rare colorectal tumor with incidence of $0.2 \%-0.6 \%$. We report a rare case of large rectal lymphoma. Our patient is a 48-year-old man, presented with 2 months history of per rectal bleed, altered bowel habits and weight loss. Clinical examination, computed tomography scan and initial endoscopic mucosal biopsy were indistinguishable from Rectal Carcinoma. With high level of suspicion, we resorted to full thickness punch biopsy in lithotomy position for a good tissue sample. Ultimately, an immunohistochemical study confirmed Diffuse Large B-cells Lymphoma (DLBCL). This case highlighted the importance of high level of suspicion for lymphoma when dealing with a rectal tumor. Accurate diagnosis of rectal lymphoma affects the treatment modalities and prognosis of the patient.
\end{abstract}

\section{Keywords}

\section{Rectal Lymphoma, Rectal Tumour, Surgery}

\section{Introduction}

Rectal lymphoma is defined as a primary extra-nodal lymphoma involving the rectum. The incidence is $0.2 \%$ $0.6 \%$ of all colorectal tumors. The rarity of rectal lymphoma and similarities of clinical presentations with rectal carcinoma contribute to a major diagnostic challenge. The distinction of rectal lymphoma from the rectal carcinoma is of vital clinical importance as the staging, management and prognosis differ from the latter. Surgery remains the mainstay of curative treatment for resectable rectal carcinoma. However, given the rarity of rectal lymphoma, the exact management remains controversial. Current guidelines are mainly based on case reports as large scale randomised controlled trials are limited. A multidisciplinary approach and individualised treatment is the key in management of rectal lymphoma. We are describing a patient with a huge rectal lymphoma in Sarawak, on the Island of Borneo.

\section{Case Report}

A 48-year-old man presented to our surgical clinic with complaints of lower abdominal pain, altered bowel habit, 
per rectal bleed, loss of weight and loss of appetite for the past two months. Patient had no significant medical history. On physical examination, he was cachexic and weak. Vital signs were stable (Temperature $36.8^{\circ} \mathrm{C}$, Pulse 76/min, Respiratory rate 22/min, Blood pressure 138/86 mmHg). Abdomen was soft and no mass was palpable. Digital rectal examination revealed a polypoidal mass with circumferential thickening located at $2 \mathrm{~cm}$ from anal verge.

Sigmoidoscopy led to the presumptive diagnosis of rectal carcinoma. However, the first endoscopic mucosal biopsy for histopathological examination was non-conclusive. It showed two tiny fragments of tissue with atypical lymphoid cells infiltrating muscularis mucosa and the affected areas were partially crushed resulting in evaluation difficulty. Hence, repetitions of endoscopic mucosal biopsy were done. This case proved to be a diagnostic challenge as the second repeated histology showed no evidence of malignancy.

With high level of suspicion, punch biopsy was done under spinal anesthesia in lithotomy position by a consultant surgeon. Adequate biopsy specimens were taken and a pathologist was consulted for immunohistochemical study. Microscopically, sections show tumor tissue exhibiting diffuse sheets of large CD20 positive lymphocytic infiltration expressing CD3-/Bcl-2+/CD10+. Mitoses are frequent consistent with high Ki67 proliferative index of $70 \%-80 \%$. Ultimately, it was confirmed as Diffuse Large B-cells Lymphoma (DLBCL). Immunohistochemical profiles favor Germinal Centre-like (GCB) molecular subtypes.

Computed tomography scan of Thorax-abdomen-pelvis (CT TAP) revealed ill-defined extensive bulky pelvic mass measuring $12.3 \times 12.8 \times 18.3 \mathrm{~cm}(\mathrm{~W} \times \mathrm{AP} \times \mathrm{CC})$ occupying nearly the entire pelvis extending up to mid abdomen (Figure 1). The mass extend superiorly to the infrarenal region and encasing the Inferior vena cava and aorta. Inferiorly, the mass encased common iliac, internal and external iliac arteries. Urinary bladder is displaced anteriorly but no invasion seen. Liver, gallbladder, spleen and adrenal glands are normal.

Routine blood tests including hemogram and renal profile were within normal limit. HIV screening was negative. In order to differentiate primary rectal lymphoma from secondary colonic involvement with non-Hodgkin Lymphoma, a further screening was done for the presence of the disease in other parts of the body. Peripheral blood film showed normal red blood cells, no blast cells and normal platelets. Bone Marrow Aspiration and trephine biopsy showed normal erythrocytic, granulocytic cells with no excess megakaryocytes, blast cells, or lymphoid cells seen.
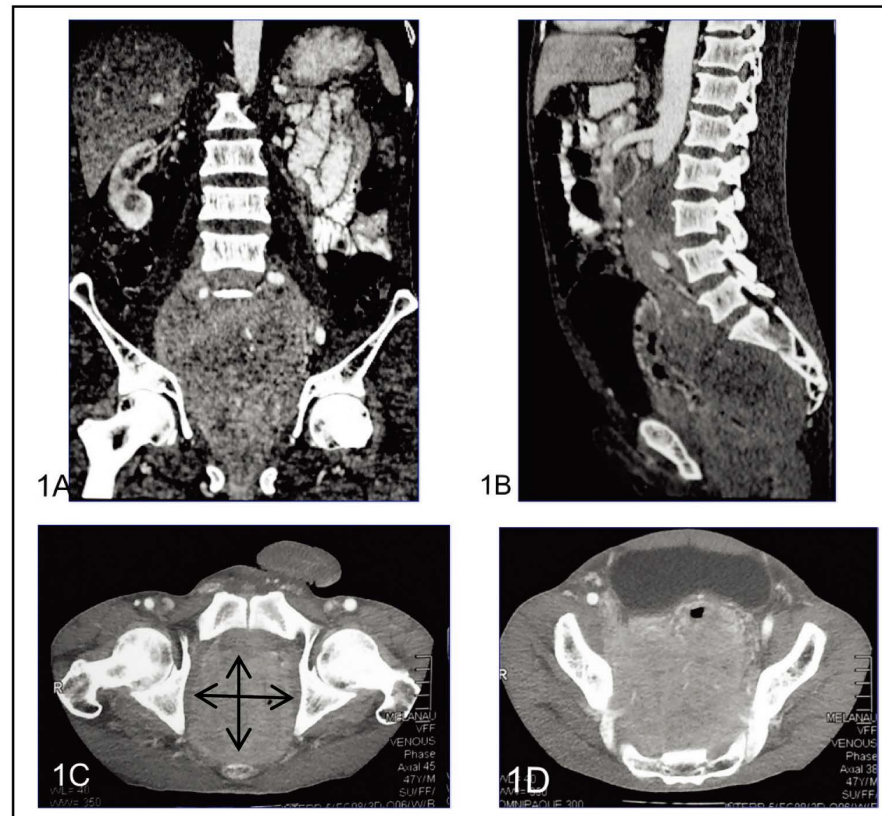

Figure 1. A Computed Tomography Scan showed (1A) Ill-defined moderately enhanching soft tissue mass occupying nearly entire pelvis extending into the mid abdomen (1B) and continue to extend superiorly to infrarenal region encasing Inferior vena cava and Aorta. Figures (1C), (1D) showed the huge mass measuring $12.3 \times 12.8 \times 18.3 \mathrm{~cm}(\mathrm{~W} \times \mathrm{AP} \times \mathrm{CC})$ and bladder is displaced anteriorly. 
This case was discussed in multidisciplinary setting (surgeon, radiologist, pathologist, oncologist and haematologist). In view of the large bulky tumor and the patient's poor premorbid, surgical resection was not thought appropriate. The patient was put on first cycle of chemotherapy (R-CHOP: rituximab, cyclophosphamide, doxorubicin, vincristine, and prednisolone). Unfortunately, the patient died after the first cycle of chemotherapy due to severe sepsis and disease progression.

\section{Discussion}

In the literature, only a few cases have been published regarding primary rectal lymphoma. Although the true aetiology has not been fully elucidated, gastrointestinal lymphoma is thought to be associated with the inflammatory bowel disease, immunodeficiency and immunosuppression [1]. However, our case involves a middle age man with no known medical illness. Our patient satisfied the five criteria of primary gastrointestinal lymphoma described by Dawson et al. [2]. These include no palpable superficial lymph node on presentation, no enlarged mediastinal nodes on chest radiograph, normal range of total and differential white cell count, involvement of only regional lymph nodes, and the liver and spleen remain without disease [2].

Despite its rarity, rectal lymphoma needs to be considered as the differential diagnosis for patients with lower gastrointestinal symptoms even without systemic features of lymphoma. In our case, the patient presented with non-specific lower abdominal pain, altered bowel habit, per rectal bleed, loss of appetite and loss of weight which is similar to that of rectal carcinoma. Wong and Eu reported cases of colorectal lymphoma with delayed diagnosis up to 10 months as the clinical presentation was non-specific abdominal pain and weight loss [3]. Clinical similarity contributes to diagnostic challenge in differentiating rectal lymphoma from rectal carcinoma. However, this case showed that computed tomography and endoscopic biopsy played important roles in differentiating rectal lymphoma from its counterpart.

Adequate knowledge on radiological features of rectal lymphoma ensures a correct diagnosis though there is no definite appearance on computed tomography. According to Fernandes $\mathrm{T}$ et al., gastrointestinal carcinoma demonstrates focal asymmetric thickening of the bowel wall on computed tomography. Meanwhile, rectal lymphoma will give long segment regular, symmetric and homogeneous wall thickening [4]. However, what is unique to rectal lymphoma is that an obstruction is uncommon due to lack of desmoplastic response and weakening of muscularispropia from submucosal lymphoid infiltration [5]. Unlike rectal carcinoma, mesorectal fatty tissue and adjacent organ involvement is less common in rectal lymphoma [6]. Apart from that, Fujii et al. demonstrated the compressibility of bowel lesion using ultrasound transducer to aid in differentiating gastrointestinal lymphoma from carcinoma. Compressibility was demonstrated in colorectal lymphoma which is absent in carcinoma [7].

Diagnosis is confirmed by histopathological examination with immunohistochemical stain. Therefore, good tissue samples of endoscopic biopsy are required. In our patient, three biopsies were attempted to reach the final diagnosis as the initial biopsies were unable to demonstrate a good sample of tissue. Bilsel Y et al. and Tan CW et al. also reported cases of failed initial biopsy as superficial biopsy of the mucosa may not provide diagnosis [8] [9]. Our case resorted to punch biopsy under spinal anesthesia which gave us a good tissue sample that showed Diffuse Large B-cells Lymphoma (DLBCL).

Treatment of rectal lymphoma remains uncertain. Management of rectal lymphoma involves multidisciplinary approach including surgery, chemotherapy and radiotherapy [3]. However, morbidity and difficulty associated with pelvic surgery for large bulky rectal lymphoma favors medical management [1]. It is difficult to suggest whether rectal lymphomas have a different behavior or if medical management alone without surgery contributes to a decreased survival time [1].

\section{Conclusion}

Primary rectal lymphoma is the rarest form of gastrointestinal lymphoma. In spite of similar presentation with rectal carcinoma, high level of suspicion, familiarity with radiological characteristic, and a good biopsy with immunohistochemical study are required for the correct diagnosis of rectal lymphoma.

\section{References}

[1] Drolet, S., Maclean, A.R., Stewart, D.A., Dixon, E., Paolucci, E.O. and Buie, W.D. (2011) Primary Colorectal Lymphoma-Clinical Outcomes in a Population-Based Series. Journal of Gastrointestinal Surgery, 15, 1851-1857. 
http://dx.doi.org/10.1007/s11605-011-1572-0

[2] Dawson, I.M., Cornes, J.S. and Morson, B.C. (1961) Primary Malignant Lymphoid Tumours of the Intestinal Tract. Report of 37 Cases with a Study of Factors Influencing Prognosis. British Journal of Surgery, 49, 80-89. http://dx.doi.org/10.1002/bjs.18004921319

[3] Wong, M.T. and Eu, K.W. (2006) Primary Colorectal Lymphoma. Colorectal Disease, 8, 586-591. http://dx.doi.org/10.1111/j.1463-1318.2006.01021.x

[4] Fernandes, T., Oliveira, M.I., Castro, R., Araújo, B., Viamonte, B. and Cunha, R. (2014) Bowel Wall Thickening at CT: Simplifying the Diagnosis. Insights Imaging, 5, 195-208. http://dx.doi.org/10.1007/s13244-013-0308-y

[5] Purysko, A.S., Coppa, C.P., Kalady, M.F., Pai, R.K., Leão Filho, H.M., Thupili, C.R. and Remer, E.M. (2014) Benign and Malignant Tumors of the Rectum and Perirectal Region. Abdominal Imaging, 4, 824-852. http://dx.doi.org/10.1007/s00261-014-0119-8

[6] Engin, G. and Korman, U. (2011) Gastrointestinal Lymphoma: A Spectrum of Fluoroscopic and CT Findings. Diagnostic and Interventional Radiology, 17, 255-265.

[7] Fujii, Y., Taniguchi, N., Koibuchi, H., Yasuda, Y. and Nagai, H. (2008) Compressibility of Gastrointestinal Tract Tumors during Transabdominal Sonographic Examination: A Clue to Diagnosis of GI Lymphoma. Journal of Clinical Ultrasound, 36, 59-62. http://dx.doi.org/10.1002/jcu.20393

[8] Bilsel, Y., Balik, E., Yamaner, S. and Bugra, D. (2005) Clinical and Therapeutic Considerations of Rectal Lymphoma: A Case Report and Literature Review. World Journal of Gastroenterology, 11, 460-461. http://dx.doi.org/10.3748/wjg.v11.i3.460

[9] Tan, C.W., Wilson, G.E., Howat, J.M. and Shreeve, D.R. (2001) Rectal Lymphoma in Ulcerative Colitis Treated with Azathioprine. European Journal of Gastroenterology and Hepatology, 13, 989-992. http://dx.doi.org/10.1097/00042737-200108000-00022 